\title{
The surgical management of obstructive hypertrophic cardiomyopathy: the RPR procedure-resection, plication, release
}

\author{
Daniel G. Swistel, Mark V. Sherrid \\ Department of Cardiothoracic Surgery and the Division of Cardiology, New York University School of Medicine, NYU/Langone Medical Center, \\ New York, NY, USA \\ Correspondence to: Daniel G. Swistel, MD. Department of Cardiothoracic Surgery, New York University School of Medicine, NYU/Langone Medical \\ Center, 530 First Avenue, New York, NY 10016, USA. Email: daniel.swistel@nyumc.org.
}

Submitted Mar 15, 2017. Accepted for publication May 10, 2017.

doi: $10.21037 /$ acs.2017.06.03

View this article at: http://dx.doi.org/10.21037/acs.2017.06.03

\section{Clinical vignette}

The patient is a 32-year-old male with a known history of hypertrophic cardiomyopathy. He had been treated for a few years with beta blockers and had good control of symptoms, but over the last year, began to be less tolerant of normal daily activities and became short of breath after meals. He also started complaining of occasional chest pain, not necessarily related to exertion. Repeat echo cardiography revealed systolic anterior motion (SAM) of the anterior leaflet of the mitral valve (ALMV) with mitral septal contact, and an outflow tract gradient of $80 \mathrm{mmHg}$ at rest. A stress echo cardiogram was obtained and the gradient increased to over $150 \mathrm{mmHg}$. The ALMV was measured at $37 \mathrm{~mm}$ from the tip of the leaflet to the insertion of the aortic cusp, including the intervalvular fibrosa. The septal thickness at the point of mitral septal contact was $24 \mathrm{~mm}$. He was started on disopyramide, which improved his shortness of breath, but he additionally complained of dry mouth and dizziness. Another repeat echo showed a persistent resting gradient $>50 \mathrm{mmHg}$. He was sent for surgical consultation and elected to proceed with surgical myectomy.

\section{Surgical technique}

In the OR, prior to incision, the intraoperative transesophageal echo cardiogram (TEE) is reviewed. Commonly, the two most useful views to confirm septal thickness, are long axis at 0 degrees (posterior septum) and 120 degrees (anterior septum). The point of mitral septal contact is identified and its location noted as distance from the aortic annulus. The length of the ALMV is reconfirmed, along with the presence of any possible accessory papillary muscle structures usually inserting as secondary attachments. Residual portions of the ALMV, commonly at the leading edge of A2, that are not involved with coaptation are also noted for possible resection. This assessment becomes more critical if the anterior septum is only modestly thickened $(<22 \mathrm{~mm})$ and truly asymmetric and isolated basally, without posterior involvement. Pre-operative MRI offers additional confirmatory information (1).

Exposure is obtained through a standard median sternotomy. Two-stage venous and retrograde coronary sinus cannulas are used and the left ventricle is vented with a $24 \mathrm{Fr}$ cannula via the right superior pulmonary vein. Moderate hypothermia (32 degrees) and blood cardioplegia are used. After cross-clamping, a transverse aortotomy above the sino-tubular junction is performed, almost transecting the aorta, as anterior retraction on the aortic annulus can be severe and tearing of the aorta is otherwise common. Leaflet retractors are used to open the aortic valve and the left ventricular chamber is examined for structures as noted above. A callus on the anterior septum is commonly seen which coincides with the point of mitral septal contact. A three-pronged hook is placed into the anterior septum beyond the callus, and using this for counter traction, a \#15 blade, on a long knife handle bent to 45 degrees, is used to start the myectomy, medially just under the right coronary ostia and proceeding laterally almost to the mitral valve. A rim of at least $5 \mathrm{~mm}$ under the aortic annulus is left behind to minimize structural collapse of the right aortic leaflet and subsequent aortic insufficiency. 
Depending on the pre-measured thickness of the septum, anywhere from 5 to $15 \mathrm{~mm}$ of muscle is resected. After the initial incision in the septum, the hook is removed and the tissue grasped with a long forcep, and with posterior displacement, the myectomy is extended to beyond the head of the antero-lateral papillary muscle. Care must be taken not to inadvertently damage papillary muscles at this stage, or to move too medially and resect posterior septum, especially if it is not hypertrophied, as a septal defect can be created here. Using this strategy, the specimen should be about $30 \mathrm{~mm}$ wide, $40 \mathrm{~mm}$ long, and the thickness will vary according to the pre-assessed available muscle tissue. A pituitary rongeur is used to remove loose pieces of muscle, smooth out irregular areas, and disconnect any lateral attachments of the antero-lateral papillary muscle to the LV free wall. Structural attachments to the ALMV can now be assessed with better visualization afforded after the myectomy. Papillary muscle heads inserting directly onto the ALMV and accessory secondary chords tenting the leaflet anteriorly can be resected if other supporting structures are present that would limit subsequent prolapse. If the ALMV is known to excessively long as the primary cause of SAM and mitral septal contact, either a horizontal plication can be performed or if a residual leaflet portion is present, it can be resected. Both these procedures require very careful pre-operative assessment and judgement. A horizontal plication with four or five horizontal mattress sutures of 5-0 proline just under the aorto-mitral curtain, can shorten an excessively long ALMV by anywhere from 3 to $6 \mathrm{~mm}$. After final inspection and irrigation and suctioning to remove any possible residual loose pieces of muscle, the aortotomy is closed with running 5-0 proline as the patient is rewarmed, and the heart allowed to fill passively with blood. Standard de-airing is carried out as the cross-clamp is removed (2). As soon as contractility is restored, the result can be assessed by either partial bypass or temporarily suspending it altogether. Adequacy of the resection and mitral insufficiency is examined. If the result is satisfactory, bypass is reinstituted, and rewarming is completed. After complete rewarming, reassessment of the repair is undertaken, an infusion of dobutamine of $10 \mathrm{mg} / \mathrm{kg}$ is started to provoke obstruction. There should no longer be any SAM, mitral septal contact or turbulent flow in the outflow tract. The residual gradient under provocation should be insignificant or at most, less than $20 \mathrm{mmHg}$. If acceptable, the dobutamine infusion is stopped, temporary pacing wires are always placed in both the right atrium and right ventricle and the operative field closed in the standard technique.

\section{Comments}

\section{Clinical results}

Our HCM center follows approximately 2,000 patients, of which over 400 have been referred for surgical management. Post-operatively, the gradients are all reduced to below $25 \mathrm{mmHg}$ on provocation, mitral insufficiency is $1+$ or trivial, and the NYHA class is $1.2 \pm 0.5$. All results have significance with $\mathrm{P}$ values of $<0.0001$. The mortality rate is $0.7 \%(n=3)$, none directly related to the myectomy procedure $(3,4)$.

\section{Advantages}

While simple myectomy, if properly performed is extremely successful in relieving outflow tract gradients and symptoms in some individuals, many obstructed patients will have persistent SAM and MR despite even a perfectly executed extended myectomy. About half of obstructed patients have very long mitral leaflets or anterior displacement of the mitral valve because of abnormal papillary muscles, or thickened, shortened chordae. Additionally, a significant proportion of patients have relatively thin septal tissue. Instead, the mitral apparatus, both leaflets and supporting structures are abnormal and must be dealt with at the time of surgery in order to resolve obstruction without resorting to mitral valve replacement. Release of antero-lateral attachments of the papillary muscle, removal or resection of accessory structures, leaflet plication and leading edge leaflet resection (residual leaflet) are all techniques that can be utilized to preserve the mitral valve (5). Additionally, they are all performed through the aortotomy used to perform the myectomy without the need for any counter incisions.

\section{Caveats}

The components of the "RPR" procedure beyond simple myectomy require experienced judgment in many instances. Although the horizontal plication is simple to perform, judging the size of the plication and suitability in individual patients may be complicated. The same is true for residual leaflet resection. The myectomy portion, if extended into 
the posterior septum, may cause a ventricular septal defect, if thickening in this area is not properly assessed preoperatively and complete heart block will invariably ensue if not performed carefully as well.

In the future, technology to better assess the intraoperative residual thickness of the septal muscle before coming off heart-lung bypass may be a way to more precisely resect the appropriate amount of tissue in cases where septal hypertrophy is limited.

\section{Acknowledgements}

None.

\section{Footnote}

Conflicts of Interest: The authors have no conflicts of interest to declare.

\section{References}

1. Sherrid MV, Chaudhry FA, Swistel DG. Obstructive

Cite this article as: Swistel DG, Sherrid MV. The surgical management of obstructive hypertrophic cardiomyopathy: the RPR procedure-resection, plication, release. Ann Cardiothorac Surg 2017;6(4):423-425. doi: 10.21037/acs.2017.06.03 hypertrophic cardiomyopathy: echocardiography, pathophysiology, and the continuing evolution of surgery for obstruction. Ann Thorac Surg 2003;75:620-32.

2. Swistel DG, DeRose JJ Jr, Sherrid MV. Management of Patients with Complex Hypertrophic Cardiomyopathy: Resection/Plication/Release. In: Cohn LH. editor. Operative Techniques in Thoracic and Cardiovascular Surgery, a comparative Atlas. Philadelphia PA: WB Saunders, 2004:261-7.

3. Swistel DG, Balaram SK. Surgical myectomy for hypertrophic cardiomyopathy in the 21 st century, the evolution of the "RPR" repair: resection, plication, and release. Prog Cardiovasc Dis 2012;54:498-502.

4. Balaram SK, Ross RE, Sherrid MV, et al. Role of mitral valve plication in the surgical management of hypertrophic cardiomyopathy. Ann Thorac Surg 2012;94:1990-7; discussion 1997-8.

5. Halpern DG, Swistel DG, Po JR, et al. Echocardiography before and after resect-plicate-release surgical myectomy for obstructive hypertrophic cardiomyopathy. J Am Soc Echocardiogr 2015;28:1318-28. 